\title{
Correlations between Endomyocardial Biopsies and Cardiac Manifestations in Taiwanese Patients with the Chinese Hotspot IVS4+919G>A Mutation: Data from the Fabry Outcome Survey
}

\author{
Ting-Rong Hsu ${ }^{1,2}$, Fu-Pang Chang ${ }^{2,3}$, Tzu-Hung Chu ${ }^{1}$, Shih-Hsien Sung ${ }^{4}$, Svetlana Bizjajeva ${ }^{5}$, \\ Wen-Chung $\mathrm{Yu}^{4, *}$ and Dau-Ming Niu ${ }^{1,2, *}$ \\ 1 Department of Pediatrics, Taipei Veterans General Hospital, Taipei 112, Taiwan; \\ romberg@gmail.com (T.-R.H.); mno4.chu@gmail.com (T.-H.C.) \\ 2 Institute of Clinical Medicine, National Yang-Ming University, Taipei 112, Taiwan; orimiltea@gmail.com \\ 3 Department of Pathology and Laboratory Medicine, Taipei Veterans General Hospital, Taipei 112, Taiwan \\ 4 Division of Cardiology, Department of Medicine, Taipei Veterans General Hospital and National Yang-Ming \\ University, Taipei 112, Taiwan; shsang@vghtpe.gov.tw \\ 5 Shire, 6300 Zug, Switzerland; sbizjajeva@shire.com \\ * Correspondence: wcyu@vghtpe.gov.tw (W.-C.Y.); dmniu1111@yahoo.com.tw (D.-M.N.); \\ Tel.: +886-2-287-121-21 (ext. 2997) (W.-C.Y.); +886-2-773-684-84 (D.-M.N.); \\ Fax: +886-2-287-717-46 (W.-C.Y.); +886-2-287-671-81 (D.-M.N.)
}

Academic Editor: Ritva Tikkanen

Received: 1 November 2016; Accepted: 21 December 2016; Published: 9 January 2017

\begin{abstract}
We retrospectively evaluated correlations between cardiac manifestations and globotriaosylceramide (Gb3) accumulation in cardiomyocytes from Taiwanese patients with Fabry disease and the IVS4+919G>A (IVS4) mutation who underwent endomyocardial biopsy (Shire; Fabry Outcome Survey data; extracted January 2015). Of 24 males and six females (median age [Q1; Q3] at biopsy 60.4 [57.4; 64.1] and 61.3 [60.4; 65.1] years, respectively), 13 males $(54.2 \%)$ and five females $(83.3 \%)$ received agalsidase alfa enzyme replacement therapy (ERT) before biopsy. Median left ventricular mass indexed to height (LVMI) within \pm 6 months of biopsy was $65.3(52.7 ; 93.1)$ in males and $53.2(42.0 ; 55.0) \mathrm{g} / \mathrm{m}^{2.7}$ in females. A moderate, positive, statistically significant correlation was found between the percentage area Gb3 accumulation in cardiomyocytes and LVMI (Spearman's $\rho$, 0.45; $p=0.014$ ); a smaller, positive, non-statistically significant correlation was observed between cardiomyocyte diameter and LVMI (Spearman's $\rho 0.16, p=0.394)$. Moderate, statistically significant, negative correlations were found between Gb3 accumulation and ERT duration (Spearman's $\rho,-0.49$, $p=0.007$ ) and between cardiomyocyte size and ERT duration (Spearman's $\rho,-0.37, p=0.048$ ). Longer ERT duration was associated with smaller amounts of Gb3 accumulation and smaller cardiomyocyte size. Further follow-up is recommended to confirm these trends in a larger sample size.
\end{abstract}

Keywords: Fabry disease; Fabry Outcome Survey; endomyocardial biopsy; enzyme replacement therapy; hypertrophic cardiomyopathy

\section{Introduction}

Progressive accumulation of glycosphingolipids, mainly globotriaosylceramide (Gb3), in cellular lysosomes throughout the body, resulting from a genetic deficiency in $\alpha$-galactosidase A activity, leads to the wide variety of signs and symptoms typically found in Fabry disease. Clinical features of classical Fabry disease tend to first appear during childhood and adolescence (e.g., acroparesthesia, pain, angiokeratomas) and progress throughout adulthood to end-stage organ disease, peaking in the fifth decade for males and the seventh for females [1]. 
Cardiac [2,3], renal [4], and cerebrovascular [5] variants of Fabry disease have been reported. In patients with the cardiac variant, disease manifestations generally occur later in life than in patients with classical Fabry disease, and are mainly restricted to the heart [2,3]. More recent reports have provided evidence indicating that these variants could actually be more common than originally thought. For example, the later-onset cardiac IVS4+919G>A (IVS4) mutation was found at a high frequency in the Taiwanese population among both newborns [6,7] and patients diagnosed with idiopathic hypertrophic cardiomyopathy [6]. However, the relationships between pathological characteristics and cardiac manifestations in patients with this later-onset variant of Fabry disease are not yet well understood.

Enzyme replacement therapy (ERT) has shown good potential in slowing the progression of, and perhaps even reversing, Fabry disease [8-10]. In Taiwan, to qualify for reimbursement for ERT, the Bureau of National Health Insurance stipulates that patients must undergo endomyocardial biopsy to confirm that Fabry disease is the main cause of their hypertrophic cardiomyopathy. This requirement provides an ideal opportunity for a closer examination of cardiac pathological changes in Taiwanese patients with the later-onset IVS4 mutation [11].

The objective of this analysis was to evaluate endomyocardial biopsy results and cardiac manifestations in Taiwanese patients with the IVS4 mutation who are registered in the Fabry Outcome Survey (FOS).

\section{Results}

As of January 2015, data were available from the FOS for 30 Taiwanese patients (24 males and six females) who had the IVS4 mutation and who also underwent endomyocardial biopsy at any point. Before biopsy, five females (83.3\%) and 13 males (54.2\%) received ERT with agalsidase alfa.

Males and females were comparable in terms of age at symptom onset, age at diagnosis, and age at start of treatment (Table 1$)$. Similarly, the median age (IQR) at biopsy was $60.4(57.4 ; 64.1)$ years in males and $61.3(60.4 ; 65.1)$ years in females (Table 1$)$. One female $(16.7 \%)$ and 10 males $(41.7 \%)$ underwent biopsy before ERT was initiated.

Table 1. Demographic characteristics and cardiac and renal parameters within \pm 6 months of the biopsy date.

\begin{tabular}{lll}
\hline $\begin{array}{l}\text { Characteristics and Parameters, } \\
\text { Median (Q1; Q3) Years }\end{array}$ & Male $(\boldsymbol{n}=\mathbf{2 4})$ & Female $(\boldsymbol{n}=\mathbf{6})$ \\
\hline Demographic Characteristics & & \\
\hline Age at symptom onset & $49.0(47.0 ; 54.0) ; n=14$ & $48.0(47.0 ; 48.0) ; n=5$ \\
Age at diagnosis & $59.0(53.5 ; 63.0)$ & $59.5(57.0 ; 62.0)$ \\
Age at FOS entry & $60.1(56.9 ; 64.2)$ & $61.1(60.1 ; 64.4)$ \\
Age at treatment start & $59.9(54.6 ; 64.2) ; n=23$ & $59.9(58.9 ; 62.9)$ \\
Age at biopsy & $60.4(57.4 ; 64.1)$ & $61.3(60.4 ; 65.1)$ \\
\hline Cardiac Parameters & & \\
\hline LVMI, g/m 2.7 & $65.3(52.7 ; 93.1) ; n=23$ & $53.2(42.0 ; 55.0)$ \\
Midwall fractional shortening, \% & $14.6(11.5 ; 16.7) ; n=20$ & $15.1(12.3 ; 18.3)$ \\
Diastolic BP, mmHg & $76.0(72.5 ; 82.0)$ & $71.0(64.0 ; 73.0) ; n=5$ \\
Systolic BP, mmHg & $120.0(115.5 ; 129.0)$ & $119.0(106.0 ; 121.0) ; n=5$ \\
MVWT, mm & $14.5(12.0 ; 18.0) ; n=23$ & $10.8(10.0 ; 13.5)$ \\
\hline Renal Parameters & & \\
\hline eGFR MDRD, mL/min $/ 1.73 \mathrm{~m}^{2}$ & $79.8(66.1 ; 92.1)$ & $73.2(55.0 ; 86.5)$ \\
Creatinine, mg/dL & $1.0(0.9 ; 1.2)$ & $0.8(0.7 ; 1.1)$ \\
\hline
\end{tabular}

$\mathrm{BP}$, blood pressure; eGFR, estimated glomerular filtration rate; FOS, Fabry Outcome Survey; LVMI, left ventricular mass indexed to height; MDRD, Modification of Diet in Renal Disease; MVWT, mean ventricular wall thickness.

Comparing cardiac parameters within \pm 6 months of the biopsy date, we found a greater median (IQR) left ventricular mass indexed to height (LVMI) of $65.3(52.7 ; 93.1) \mathrm{g} / \mathrm{m}^{2.7}$ in males compared 
with $53.2(42.0 ; 55.0) \mathrm{g} / \mathrm{m}^{2.7}$ in females (Table 1 ; one male did not have LVMI data within \pm 6 months of the biopsy date and was excluded from this analysis). Median ventricular wall thickness was also greater in males than females $(14.5[12.0 ; 18.0]$ vs. $10.8[10.0 ; 13.5] \mathrm{mm})$, as was the median diastolic blood pressure (BP) $(76.0$ [72.5; 82.0] vs. 71.0 [64.0; 73.0] mmHg; Table 1). Systolic BP and midwall fractional shortening were similar in both sexes (Table 1 ).

Regarding renal parameters within \pm 6 months of the biopsy date, the median (IQR) estimated glomerular filtration rate (eGFR) using the Modification of Diet in Renal Disease (MDRD) was slightly greater in males than females $\left(79.8[66.1 ; 92.1]\right.$ vs. $\left.73.2[55.0 ; 86.5] \mathrm{mL} / \mathrm{min} / 1.73 \mathrm{~m}^{2}\right)$, whereas the creatinine level was similar in both sexes (Table 1).

The median (IQR) area of Gb3 accumulation in cardiomyocytes was similar in males and females $(0.10 \%[0.06 \% ; 0.12 \%]$ vs. $0.05 \%[0.03 \% ; 0.11 \%])$. Furthermore, no difference was observed in the median cardiomyocyte size between males and females $(24.1$ [23.2; 26.1] vs. $24.1[23.5 ; 25.8] \mu \mathrm{m})$.

A positive correlation was found between the percentage area of Gb3 accumulation within cardiomyocytes and LVMI in our overall IVS4 population (Spearman's correlation coefficient 0.45 , $p=0.014$; Figure 1a). A positive correlation of a smaller size was observed between the cardiomyocyte size and LVMI (Spearman's correlation coefficient $0.16, p=0.394$; Figure 1b), although this correlation was not statistically significant.

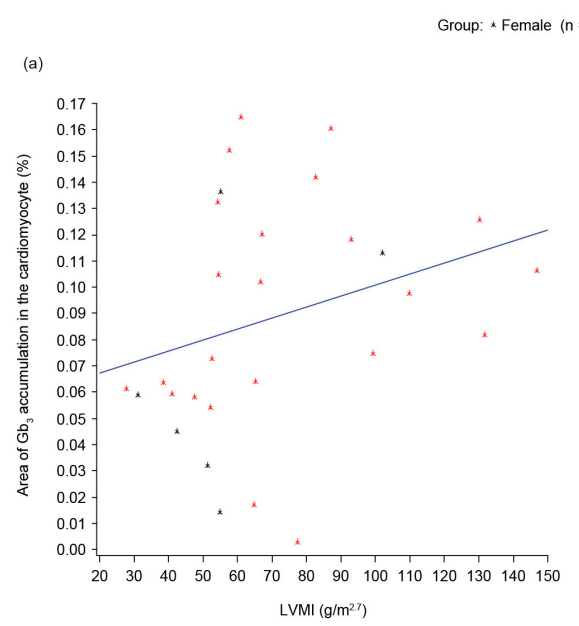

Male $(n=23)$

(b)
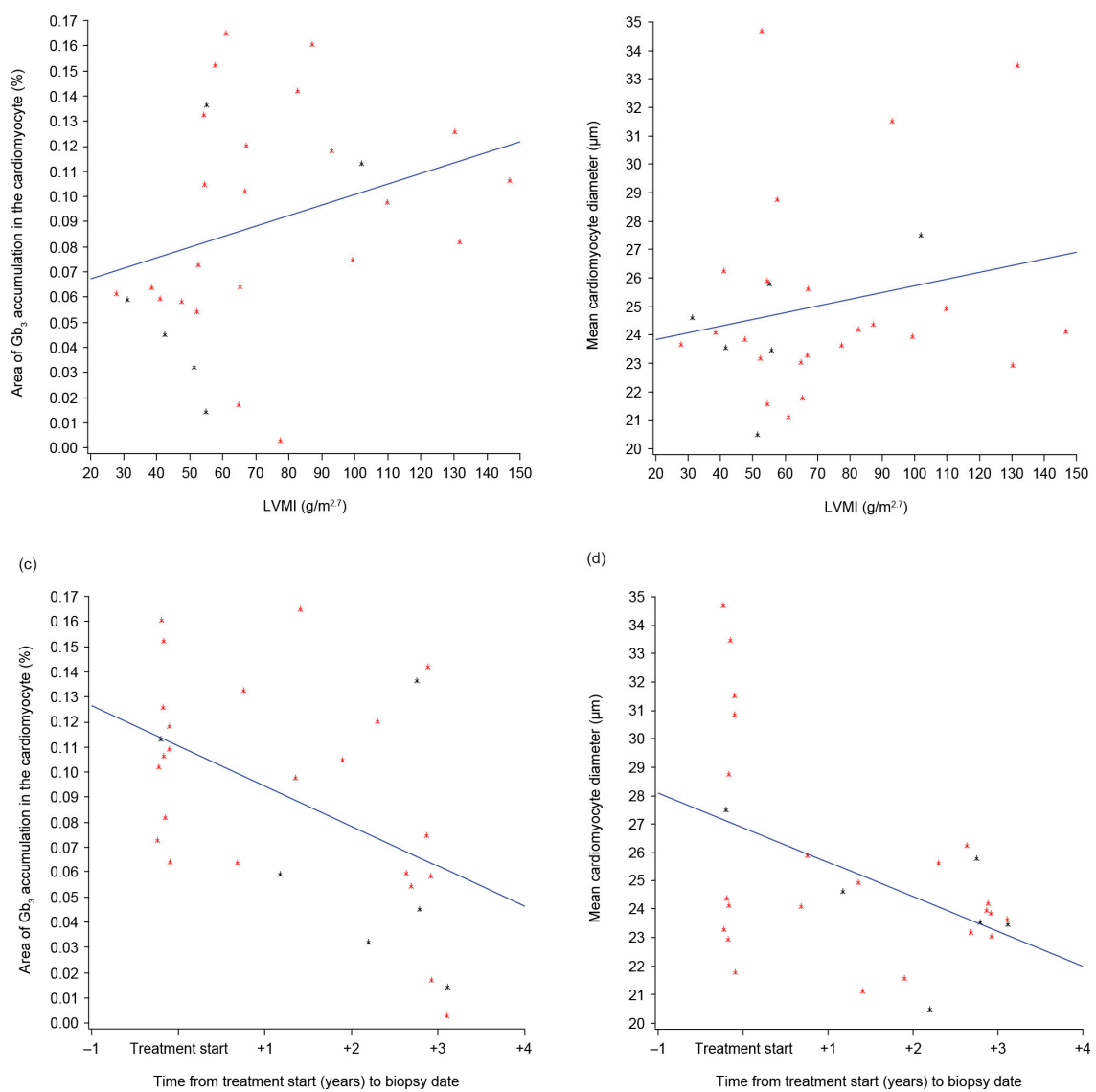

Figure 1. Scatter plots illustrating: (a) Globotriaosylceramide (Gb3) accumulation by left ventricular mass indexed to height (LVMI); (b) Cardiomyocyte size by LVMI; (c) Gb3 accumulation by time from treatment start to biopsy date; (d) Cardiomyocyte size by time from treatment start to biopsy date. Left ventricular hypertrophy was defined as LVMI of $>51 \mathrm{~g} / \mathrm{m}^{2.7}$ in males and $>48 \mathrm{~g} / \mathrm{m}^{2.7}$ in females [12]. 
A negative correlation was found between Gb3 accumulation and the duration of ERT (Spearman's correlation coefficient $-0.49, p=0.007$; Figure 1c) and also between the cardiomyocyte size and the duration of ERT (Spearman's correlation coefficient $-0.37, p=0.048$; Figure 1d), both of which were statistically significant.

\section{Discussion}

This is the first study comparing endomyocardial biopsy results and cardiac parameters in Fabry disease. We found a statistically significant positive correlation between the area of Gb3 accumulation within cardiomyocytes and LVMI; a similar positive correlation was also observed between cardiomyocyte size and LVMI, although this was not statistically significant. We also found negative correlations between cardiomyocyte size and the time from the start of treatment, and between Gb3 accumulation and the time from the start of treatment, both of which were statistically significant. The latter observation is in agreement with results from our previous analysis, in which a relatively small amount of Gb3 accumulation was observed in the cardiomyocytes of patients who had received ERT for the longest duration [11]. Despite reports of limited Gb3 clearance from cardiomyocytes in patients receiving ERT $[13,14]$, our findings could provide further evidence to suggest that ERT may be beneficial in patients with later-onset Fabry disease.

Histological findings of Gb3 deposition in the cardiomyocytes can be an early indicator of cardiac involvement before signs and symptoms manifest, particularly left ventricular hypertrophy [11,15]. Earlier diagnosis and treatment initiation are expected to have beneficial consequences for the management of classical Fabry disease, and also for the later-onset cardiac variant [16].

Regarding the mechanism of hypertrophic cardiomyopathy in Fabry disease, it has been hypothesized that the accumulation of Gb3 triggers a number of pathophysiological processes, such as inflammatory reactions, which, when combined with the activity of growth-promoting factors such as plasma globotriaosylsphingosine and sphingosine-1-phosphate, or other genetic or environmental factors, may result in progressive cardiovascular remodeling $[17,18]$. Thus, it is likely that Gb3 accumulation is the main trigger of cardiac hypertrophy, but not its main cause. Consistent with this hypothesis, in our study, the percentage area of Gb3 accumulation was only around $0.1 \%-0.2 \%$ of the area of the cardiomyocytes.

This analysis is retrospective and uses data from the FOS registry, which was not specifically designed to collect data on the parameters investigated herein; furthermore, the inclusion criteria are less rigid than would be required for a clinical trial. The small sample size, particularly for females, is a further limiting factor and means that the statistical analysis must be considered exploratory and descriptive.

We have previously reported results from endomyocardial biopsies in Taiwanese patients that strongly supported the role of IVS4 as a pathogenic later-onset Fabry mutation [11], and the results herein provide further evidence to support this. Further follow-up analyses and the collection of additional data are required to confirm the trends reported here and to increase the knowledge base of cardiac pathology in later-onset IVS4 Fabry disease.

\section{Materials and Methods}

\subsection{Patients and Study Design}

This was a retrospective analysis of data entered in FOS, an international outcomes database sponsored by Shire for the collection of data on the natural course and treatment of Fabry disease. Patients with confirmed Fabry disease who are receiving, or are candidates for, ERT with agalsidase alfa are eligible for inclusion in FOS. All procedures followed are in accordance with the ethical standards of the responsible committees on human experimentation (institutional and national) and with the Helsinki Declaration of 1975, as revised in 2000. The participation of each center in FOS has 
been approved by the respective ethics committees or institutional review boards. All patients gave written, informed consent before their data were entered into FOS.

The inclusion criteria for this analysis were Taiwanese patients who carried the Chinese hotspot IVS4+919G>A mutation, as confirmed by molecular analysis, who also underwent endomyocardial biopsy at any point.

The FOS data used for this analysis were extracted from the FOS database in January 2015.

\subsection{Clinical Assessments}

Data from all routine clinical assessments undertaken at each visit are entered into the FOS database but, for the purposes of this analysis, only values closest to the biopsy date (within \pm 6 months) for the following assessments were included: diastolic and systolic BP, eGFR using the MDRD equation, and serum creatinine.

Echocardiography to determine left ventricular mass indexed to height (LVMI $\mathrm{g} / \mathrm{m}^{2.7}$ ) was performed according to prespecified FOS guidelines and using recommendations published by the American Society of Echocardiography [19]. Additional echocardiographic measurements included midwall fractional shortening and ventricular wall thickness. Only values closest to the biopsy date (within \pm 6 months) were analyzed.

\subsection{Cardiac Pathology}

Endomyocardial biopsy using a flexible endomyocardial bioptome was performed to obtain endomyocardial tissue from the right side of the interventricular septum. Then, cardiomyocyte size and percentage area of Gb3 accumulation were assessed in cardiomyocytes from these endomyocardial tissues.

Cardiomyocyte diameter was obtained from transverse sections by measuring the minor axis at the nuclear level, as performed in our previous study [11]. A total of 15 cells from three micrographs were measured for each sample.

The average percentage area of Gb3 accumulation in cardiomyocytes on toluidine blue stained micrographs was obtained using ImageJ software, an image processing program developed by the US National Institutes of Health (Bethesda, MD, USA) (available for download at http://rsb.info.nih.gov/ij/).

The total area of cardiomyocytes within a micrograph (Figure 2a) was obtained by adjusting the color threshold tool to select and measure the desired area (Figure 2b). The color threshold tool was then used to select areas of toluidine blue staining to measure the area of $\mathrm{Gb} 3$ accumulation within the cardiomyocytes (Figure 2c). The percentage of Gb3 deposition was calculated within ImageJ using these two values. All histopathological specimens were reviewed by the same pathologist, who was blinded to the clinical information.

(a)

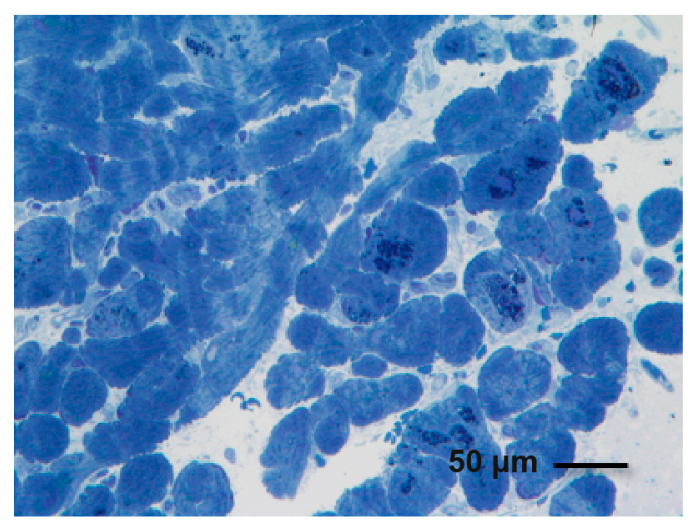

Figure 2. Cont. 
(b)

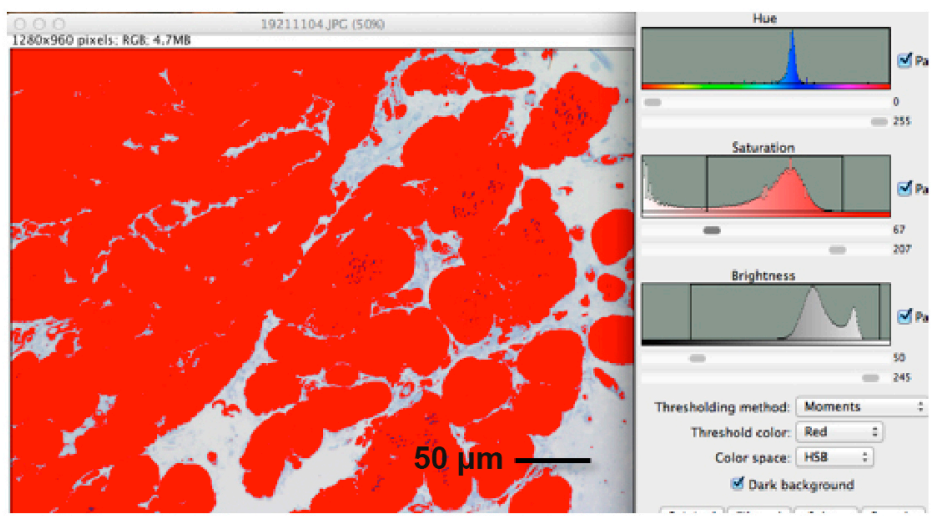

(c)

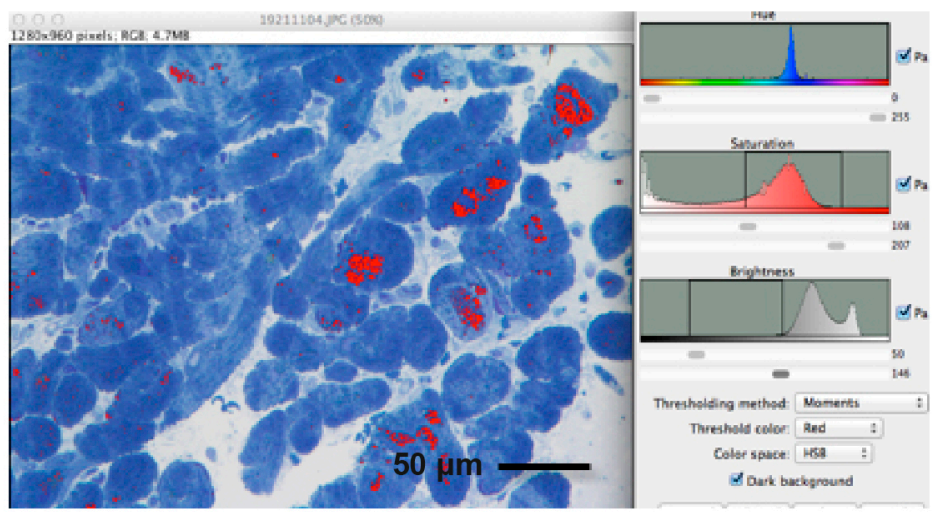

Figure 2. Image processing using ImageJ software. (a) Cardiomyocyte sample from a single Fabry patient with toluidine blue staining; (b) Total area of the cardiomyocytes (in red); (c) Area of globotriaosylceramide (Gb3) deposition within the cardiomyocytes. The percentage area of Gb3 deposition was calculated from these two values, using ImageJ.

\subsection{Statistical Analysis}

Due to the small sample size, non-parametric measures were used. Descriptive statistics were calculated for the demographic and clinical characteristics. Spearman's rank correlation coefficient was calculated to assess the relationship between cardiac parameters and cardiac biopsy results. Statistical significance was set at 5\%, and SAS software Version 9.2 (SAS Institute Inc., Cary, NC, USA) was used.

\section{Conclusions}

The correlation between LVMI and Gb3 accumulation in cardiomyocytes was moderate but statistically significant in our IVS4 patients. Furthermore, in the patients treated with ERT, both Gb3 accumulation and cardiomyocyte size showed moderate, statistically significant, negative correlations with time from the start of treatment to the biopsy date; thus, longer treatment periods were associated with smaller amounts of Gb3 accumulation and smaller cardiomyocyte size.

Acknowledgments: The Fabry Outcome Survey is sponsored by Shire Human Genetic Therapies, Inc. Shire International $\mathrm{GmbH}$ provided funding to Excel Scientific Solutions for support in writing and editing this manuscript. Under the direction of the authors, Tina Rose, an employee of Excel Scientific Solutions, provided writing assistance for this publication. Editorial assistance in formatting, proofreading, copy editing, and fact checking was also provided by Excel Scientific Solutions. Svetlana Bizjajeva from Shire International GmbH also reviewed and edited the manuscript for scientific accuracy. Amandine Perrin, a former employee of Shire International $\mathrm{GmbH}$, contributed to the initial data analysis. 
Author Contributions: Dau-Ming Niu, Ting-Rong Hsu, and Wen-Chung Yu designed the study; Svetlana Bizjajeva performed the statistical analysis; Ting-Rong Hsu, Fu-Pang Chang, Tzu-Hung Chu, Shih-Hsien Sung, Svetlana Bizjajeva, Wen-Chung Yu, and Dau-Ming Niu interpreted the data, drafted and critically reviewed the manuscript, and approved the final version for submission.

Conflicts of Interest: Ting-Rong Hsu has received travel support from Genzyme and Shire. Svetlana Bizjajeva is an employee of, and holds stock options in, Shire. Wen-Chung Yu has received travel support from Genzyme and Shire. Dau-Ming Niu has received research and travel support from Genzyme and Shire. Fu-Pang Chang, Tzu-Hung Chu, and Shih-Hsien Sung declare no conflicts of interest.

\section{References}

1. Mehta, A.; Widmer, U. Natural history of Fabry disease. In Fabry Disease: Perspectives from 5 Years of FOS; Mehta, A., Beck, M., Sunder-Plassmann, G., Eds.; Oxford PharmaGenesis: Oxford, UK, 2006; pp. 183-188.

2. Von Scheidt, W.; Eng, C.M.; Fitzmaurice, T.F.; Erdmann, E.; Hübner, G.; Olsen, E.G.; Christomanou, H.; Kandolf, R.; Bishop, D.F.; Desnick, R.J. An atypical variant of Fabry's disease with manifestations confined to the myocardium. N. Engl. J. Med. 1991, 324, 395-399. [CrossRef] [PubMed]

3. Nakao, S.; Takenaka, T.; Maeda, M.; Kodama, C.; Tanaka, A.; Tahara, M.; Yoshida, A.; Kuriyama, M.; Hayashibe, H.; Sakuraba, H.; et al. An atypical variant of Fabry's disease in men with left ventricular hypertrophy. N. Engl. J. Med. 1995, 333, 288-293. [CrossRef] [PubMed]

4. Nakao, S.; Kodama, C.; Takenaka, T.; Tanaka, A.; Yasumoto, Y.; Yoshida, A.; Kanzaki, T.; Enriquez, A.L.; Eng, C.M.; Tanaka, H.; et al. Fabry disease: Detection of undiagnosed hemodialysis patients and identification of a "renal variant" phenotype. Kidney Int. 2003, 64, 801-807. [CrossRef] [PubMed]

5. Rolfs, A.; Böttcher, T.; Zschiesche, M.; Morris, P.; Winchester, B.; Bauer, P.; Walter, U.; Mix, E.; Lohr, M.; Harzer, K.; et al. Prevalence of Fabry disease in patients with cryptogenic stroke: A prospective study. Lancet 2005, 366, 1794-1796. [CrossRef]

6. Lin, H.Y.; Chong, K.W.; Hsu, J.H.; Yu, H.C.; Shih, C.C.; Huang, C.H.; Lin, S.J.; Chen, C.H.; Chiang, C.C.; Ho, H.J.; et al. High incidence of the cardiac variant of Fabry disease revealed by newborn screening in the Taiwan Chinese population. Circ. Cardiovasc. Genet. 2009, 2, 450-456. [CrossRef] [PubMed]

7. Hwu, W.L.; Chien, Y.H.; Lee, N.C.; Chiang, S.C.; Dobrovolny, R.; Huang, A.C.; Yeh, H.Y.; Chao, M.C.; Lin, S.J.; Kitagawa, T.; et al. Newborn screening for Fabry disease in Taiwan reveals a high incidence of the later-onset GLA mutation c.936+919G>A (IVS4+919G>A). Hum. Mutat. 2009, 30, 1397-1405. [CrossRef] [PubMed]

8. Mehta, A.; West, M.L.; Pintos-Morell, G.; Reisin, R.; Nicholls, K.; Figuera, L.E.; Parini, R.; Carvalho, L.R.; Kampmann, C.; Pastores, G.M.; et al. Therapeutic goals in the treatment of Fabry disease. Genet. Med. 2010, 12, 713-720. [CrossRef] [PubMed]

9. Weidemann, F.; Niemann, M.; Breunig, F.; Herrmann, S.; Beer, M.; Stork, S.; Voelker, W.; Ertl, G.; Wanner, C.; Strotmann, J. Long-term effects of enzyme replacement therapy on fabry cardiomyopathy: Evidence for a better outcome with early treatment. Circulation 2009, 119, 524-529. [CrossRef] [PubMed]

10. Germain, D.P.; Charrow, J.; Desnick, R.J.; Guffon, N.; Kempf, J.; Lachmann, R.H.; Lemay, R.; Linthorst, G.E.; Packman, S.; Scott, C.R.; et al. Ten-year outcome of enzyme replacement therapy with agalsidase $\beta$ in patients with Fabry disease. J. Med. Genet. 2015, 52, 353-358. [CrossRef] [PubMed]

11. Hsu, T.R.; Sung, S.H.; Chang, F.P.; Yang, C.F.; Liu, H.C.; Lin, H.Y.; Huang, C.K.; Gao, H.J.; Huang, Y.H.; Liao, H.C.; et al. Endomyocardial biopsies in patients with left ventricular hypertrophy and a common Chinese later-onset Fabry mutation (IVS4+919G>A). Orphanet J. Rare Dis. 2014, 9, 96. [CrossRef] [PubMed]

12. De Simone, G.; Daniels, S.R.; Devereux, R.B.; Meyer, R.A.; Roman, M.J.; de Divitiis, O.; Alderman, M.H. Left ventricular mass and body size in normotensive children and adults: Assessment of allometric relations and impact of overweight. J. Am. Coll. Cardiol. 1992, 20, 1251-1260. [CrossRef]

13. Pieroni, M. Echocardiographic assessment of Fabry cardiomyopathy: Early diagnosis and follow-up. J. Am. Soc. Echocardiogr. 2011, 24, 1033-1036. [CrossRef] [PubMed]

14. Thurberg, B.L.; Fallon, J.T.; Mitchell, R.; Aretz, T.; Gordon, R.E.; O'Callaghan, M.W. Cardiac microvascular pathology in Fabry disease: Evaluation of endomyocardial biopsies before and after enzyme replacement therapy. Circulation 2009, 119, 2561-2567. [CrossRef] [PubMed]

15. Tschöpe, C.; Dominguez, F.; Canaan-Kühl, S.; Blaschke, D.; Kühl, U.; Pieske, B.; Haverkamp, W. Endomyocardial biopsy in Anderson-Fabry disease: The key in uncertain cases. Int. J. Cardiol. 2015, 190, 284-286. [CrossRef] [PubMed] 
16. Biegstraaten, M.; Arngrimsson, R.; Barbey, F.; Boks, L.; Cecchi, F.; Deegan, P.B.; Feldt-Rasmussen, U.; Geberhiwot, T.; Germain, D.P.; Hendriksz, C.; et al. Recommendations for initiation and cessation of enzyme replacement therapy in patients with Fabry disease: The European Fabry Working Group consensus document. Orphanet J. Rare Dis. 2015, 10, 36. [CrossRef] [PubMed]

17. Seydelmann, N.; Wanner, C.; Stork, S.; Ertl, G.; Weidemann, F. Fabry disease and the heart. Best Pract. Res. Clin. Endocrinol. Metab. 2015, 29, 195-204. [CrossRef] [PubMed]

18. Hsu, T.R.; Hung, S.C.; Chang, F.P.; Yu, W.C.; Sung, S.H.; Hsu, C.L.; Dzhagalov, I.; Yang, C.F.; Chu, T.H.; Lee, H.J.; et al. Later onset Fabry disease, cardiac damage progress in silence: Experience with a highly prevalent mutation. J. Am. Coll. Cardiol. 2016, 68, 2554-2563. [CrossRef] [PubMed]

19. Lang, R.M.; Bierig, M.; Devereux, R.B.; Flachskampf, F.A.; Foster, E.; Pellikka, P.A.; Picard, M.H.; Roman, M.J.; Seward, J.; Shanewise, J.S.; et al. Recommendations for chamber quantification: A report from the American Society of Echocardiography's Guidelines and Standards Committee and the Chamber Quantification Writing Group, developed in conjunction with the European Association of Echocardiography, a branch of the European Society of Cardiology. J. Am. Soc. Echocardiogr. 2005, 18, 1440-1463. [PubMed]

(c) 2017 by the authors; licensee MDPI, Basel, Switzerland. This article is an open access article distributed under the terms and conditions of the Creative Commons Attribution (CC-BY) license (http://creativecommons.org/licenses/by/4.0/). 